\title{
STRONG AND WEAK WEIGHTED NORM INEQUALITIES FOR THE GEOMETRIC FRACTIONAL MAXIMAL OPERATOR
}

\author{
SORINA BARZA ${ }^{\bowtie}$ and CONSTANTIN P. NICULESCU
}

(Received 27 September 2011)

\begin{abstract}
We characterise the strong- and weak-type boundedness of the geometric fractional maximal operator between weighted Lebesgue spaces in the case $0<p \leq q<\infty$, generalising and improving some older results.
\end{abstract}

2010 Mathematics subject classification: primary 42B25; secondary $26 \mathrm{D} 15$.

Keywords and phrases: geometrical maximal operator, weighted Lebesgue space, strong-/weak-type inequality.

\section{Introduction}

The purpose of this paper is to prove necessary and sufficient conditions on the weights for which the geometric fractional maximal operator of order $\alpha \in[0, n)$,

$$
\begin{aligned}
G_{\alpha} f(x) & =\sup _{Q \in \mathfrak{C}_{x}}|Q|^{\alpha / n} \exp \left(\frac{1}{|Q|} \int_{Q} \log |f(y)| d y\right) \\
& =\sup _{Q \in \mathfrak{C}_{x}} \lim _{\gamma \rightarrow 0^{+}}|Q|^{\alpha / n}\left(\frac{1}{|Q|} \int_{Q}|f(y)|^{\gamma} d y\right)^{1 / \gamma},
\end{aligned}
$$

satisfies the two-weight strong-type or weak-type $\left(L^{p}(v), L^{q}(u)\right)$ estimates. Here, $\mathfrak{C}_{x}$ denotes the family of all cubes $Q \subset \mathbb{R}^{n}$ containing $x$, and $f$ is a measurable function on $\mathbb{R}^{n}$. The sides of the cubes in $\mathfrak{C}_{x}$ are assumed to be parallel to the coordinate axes. For $r>0, r Q$ will denote the cube with centre that of $Q$ and diameter $r$ times that of $Q$.

The operator $G_{\alpha}$ is a submultiplicative analogue of the usual fractional maximal operator of order $\alpha$,

$$
M_{\alpha} f(x)=\sup _{Q \in \mathfrak{C}_{x}}|Q|^{\alpha / n-1} \int_{Q}|f(y)| d y,
$$

(C) 2012 Australian Mathematical Publishing Association Inc. 0004-9727/2012 \$16.00 
which in turn is a generalisation of the classical Hardy-Littlewood maximal operator,

$$
M f(x)=\sup _{Q \in \mathfrak{C}_{x}} \frac{1}{|Q|} \int_{Q} f(y) d y .
$$

Weighted norm inequalities for the maximal and the fractional maximal operator have been extensively studied (see, for example, $[4,5,7]$ and the references therein), while their submultiplicative analogues have come to attention more recently. See, for example, $[1-3,9]$ for results and historical facts.

By Jensen's inequality,

$$
G_{\alpha} f(x) \leq M_{\alpha} f(x)
$$

and strict inequalities are possible. Another simple but useful property of the geometric fractional maximal operator is

$$
G_{\alpha+\beta} f g \leq G_{\alpha} f G_{\beta} g \text { for } \alpha, \beta \geq 0, \alpha+\beta<n .
$$

If $\alpha=\beta=0$, the above relation expresses the submultiplicativity of the geometric maximal operator. We also have $G_{\alpha} a f^{p}=a\left(G_{\alpha / p} f\right), a \in \mathbb{R}, p>0$.

In this paper we are interested in the action of $G_{\alpha}$ on weighted Lebesgue spaces. By a weight we will mean any function which is locally integrable and positive on $\mathbb{R}^{n}$. Given a weight $u$ and an exponent $p>0$ the symbols $L^{p}(u)$ and $L^{p, \infty}(u)$ will denote the classical weighted strong- and weak-type Lebesgue spaces on $\mathbb{R}^{n}$ with the Lebesgue measure, with the usual modification if $p=\infty$. If $E$ is a Lebesgue measurable subset of $\mathbb{R}^{n}$ then $|E|$ will denote the Lebesgue measure of $E$. The letter $C$ will be used to denote a positive constant not necessarily the same at each occurrence.

In Section 2 we characterise the two-weight strong-type inequality for the geometric fractional maximal operator $G_{\alpha}$. The special case $n=1, \alpha=0, p=q$ was completely characterised in [9]. The case $n>1, \alpha=0, p=q$ was considered in [1] but some restrictive conditions were a priori assumed on the weight on the right-hand side. The technique used there was to treat the geometrical operator as a limit of minimal operators. The complete characterisation of the strong-type inequality for the geometrical fractional maximal operator (that is, $\alpha \neq 0$ ) in the one-dimensional case $n=1,0<p \leq q<\infty$ was given in [3]. Using techniques of more classical character (see, for example, [4]) we extend the previous results to the case $n>1, \alpha \neq 0$ and $0<p \leq q<\infty$. Although we also assume an extra condition on the weight on the righthand side, it seems to be easier to verify than that imposed in [1]. In the diagonal case, that is, when the weights are equal, we show that the a priori condition is redundant (see Corollary 2.4).

In Section 3 we characterise (assuming only the doubling condition on the weight on the left-hand side) the corresponding two-weight weak-type inequality for the geometric fractional maximal operator $G_{\alpha}$, for $n>1, \alpha \neq 0,0<p \leq q<\infty$. This result extends to the multidimensional case the corresponding results proved in [3, Theorem 1] and [9, Theorem 2]. The case $\alpha=0, n>1, p=q$ was considered 
in [1] where more complicated conditions were assumed on the weights. In the oneweighted case we do not have to assume any a priori conditions on the weights (see Corollary 3.4). In this way we answer an open question posed in [1].

\section{Strong-type weighted inequalities}

The aim of this section is to prove necessary and sufficient conditions under which the two-weight norm inequality for the geometric fractional maximal operator holds true, that is, $G_{\alpha}: L^{p}(v) \rightarrow L^{q}(u)$. The proof of our main result is based on the following lemma.

Lemma 2.1. Let $v$ be a weight function with a positive lower bound, $Q \subset \mathbb{R}^{n}$ a cube with sides parallel to the coordinate axes and $\alpha \geq 0$. Suppose that $f$ is a positive, bounded function with compact support such that

$$
|Q|^{\alpha / n} \exp \left(\frac{1}{|Q|} \int_{Q} \log f(x) d x\right)>t, \quad t>0 .
$$

Then there exists a dyadic cube $P$ (which intersects $Q$ ) such that

$$
\left(\frac{1}{|P|} \int_{P} f(x) v^{1 / p}(x) d x\right)\left(|Q|^{\alpha / n} \exp \left(\frac{1}{|Q|} \int_{Q} \log v^{-1 / p}(x) d x\right)\right)>\frac{1}{2^{2 n}} t, \quad t>0 .
$$

Proof. The restrictions on $f$ and $v$ ensure that a general equality of the form

$$
\exp \left(\int_{Q} \log |f|\right)=\exp \left(\int_{Q} \log |f| h\right) \exp \left(\int_{Q} \log \frac{1}{h}\right)
$$

holds (see for example [9, Theorem 1]). Let $k \in \mathbb{Z}$ such that $2^{(k-1) n} \leq|Q|<2^{k n}$. Then there exist $P_{1}, \ldots, P_{N}\left(1<N \leq 2^{n}\right)$, dyadic cubes of generation $2^{k}$ which intersect $Q$, such that $Q \subset \bigcup_{j=1}^{N} P_{j}$. Clearly, $Q \subset 3 P_{j}$ for any $j$. There exists at least one dyadic cube $P$ (among $P_{1}, \ldots, P_{N}$ ) such that

$$
\left(\frac{1}{|Q|} \int_{P} f(x) v^{1 / p}(x) d x\right)\left(|Q|^{\alpha / n} \exp \left(\frac{1}{|Q|} \int_{Q} \log v^{-1 / p}(x) d x\right)\right)>\frac{1}{2^{n}} t, \quad t>0 .
$$

If this is not true, then, by Jensen's inequality,

$$
\begin{aligned}
|Q|^{\alpha / n} & \exp \left(\frac{1}{|Q|} \int_{Q} \log f(x) d x\right) \\
& \leq\left(\frac{1}{|Q|} \int_{Q} f(x) v^{1 / p}(x) d x\right)\left(|Q|^{\alpha / n} \exp \left(\frac{1}{|Q|} \int_{Q} \log v^{-1 / p}(x) d x\right)\right) \\
& \leq\left(\frac{1}{|Q|} \sum_{j=1}^{N} \int_{P_{j}} f(x) v^{1 / p}(x) d x\right)\left(|Q|^{\alpha / n} \exp \left(\frac{1}{|Q|} \int_{Q} \log v^{-1 / p}(x) d x\right)\right) \\
& \leq \frac{N t}{2^{n}} \leq t
\end{aligned}
$$


which contradicts the hypothesis (2.1). Hence, by (2.1),

$$
\begin{aligned}
& \left(\frac{1}{|P|} \int_{P} f(x) v^{1 / p}(x) d x\right)\left(|Q|^{\alpha / n} \exp \left(\frac{1}{|Q|} \int_{Q} \log v^{-1 / p}(x) d x\right)\right) \\
& \quad=\frac{|Q|}{|P|}\left(\frac{1}{|Q|} \int_{P} f(x) v^{1 / p}(x) d x\right)\left(|Q|^{\alpha / n} \exp \left(\frac{1}{|Q|} \int_{Q} \log v^{-1 / p}(x) d x\right)\right)>\frac{1}{2^{2 n}} t
\end{aligned}
$$

and this completes the proof.

The following theorem, which is the main result of this section, characterises the strong boundedness of the geometrical fractional maximal operator.

Theorem 2.2. Let $0<p \leq q<\infty, 0 \leq \alpha<n$ and $P$ be a dyadic cube. Suppose that

$$
\frac{1}{|3 P|} \int_{3 P} \log v(x) d x \leq \frac{1}{|Q|} \int_{Q} \log K v(x) d x
$$

for some $K>0$ and any cube $Q \subset 3 P$ which intersects $P$ and such that $\left(1 / 2^{n}\right)|P| \leq$ $|Q|<|P|$. Then there exists a constant $C$ such that the inequality

$$
\left(\int_{\mathbb{R}^{n}}\left(G_{\alpha}|f(x)|\right)^{q} u(x) d x\right)^{1 / q} \leq C\left(\int_{\mathbb{R}^{n}}|f|^{p}(x) v(x) d x\right)^{1 / p}
$$

holds for all $f \in L^{p}(v)$ if and only if there exists a constant $C$ such that,

$$
\left(\int_{Q}\left(G_{\alpha p}\left(\chi_{Q} v^{-1}\right)\right)^{q / p} u(x) d x\right)^{1 / q}<C|Q|^{1 / p}
$$

for every cube $Q \subset \mathbb{R}^{n}$ with sides parallel to the coordinate axes.

Proof. The necessity part follows from (2.3), applied to $f=\chi_{Q} v^{-1 / p}$.

The rest of the proof will concern the sufficiency of the condition (2.4) for the norm inequality (2.3) to hold. We may assume, by standard arguments, that $f \in L^{p}(v)$ is a nonnegative function with compact support and that $v$ has the lower bound 1 (see, for example, [9]). Write

$$
\Omega_{k}=\left\{x \in \mathbb{R}^{n}: 2^{k}<G_{\alpha} f(x) \leq 2^{k+1}\right\} .
$$

By the definition of $G_{\alpha}$, for every $k \in \mathbb{Z}$ and every $x \in \Omega_{k}$, there exists a cube $Q_{x}^{k}$ such that

$$
\left|Q_{x}^{k}\right|^{\alpha / n} \exp \left(\frac{1}{\left|Q_{x}^{k}\right|} \int_{Q_{x}^{k}} \log f(x) d x\right)>2^{k} .
$$

By Lemma 2.1 we can find a dyadic cube $P_{x}^{k}$ which intersects $Q_{x}^{k}$, with $Q_{x}^{k} \subset 3 P_{x}^{k}$ and such that

$$
\left(\frac{1}{\left|P_{x}^{k}\right|} \int_{P_{x}^{k}} f(x) v^{1 / p}(x) d x\right)\left(\left|Q_{x}^{k}\right|^{\alpha / n} \exp \left(\frac{1}{\left|Q_{x}^{k}\right|} \int_{Q_{x}^{k}} \log v^{-1 / p}(x) d x\right)\right)>\frac{1}{2^{2 n}} 2^{k}
$$


This estimate and the fact that $f \in L^{p}(v)$ yield that the dyadic cubes $P_{x}^{k}$ have bounded size, for every $k$. Hence there exists a sequence of maximal (and so disjoint) dyadic cubes $\left\{P_{j}^{k}\right\}_{j \in \mathbb{N}}$ such that every $Q_{x}^{k} \subset 3 P_{j}^{k}$, for some $j \in \mathbb{N}$. As a consequence,

$$
\Omega_{k} \subset \bigcup_{j \in \mathbb{N}} 3 P_{j}^{k} .
$$

We now decompose $\Omega_{k}$ in the standard way:

$$
E_{1}^{k}=3 P_{1}^{k} \cap \Omega_{k}, E_{2}^{k}=\left(3 P_{2}^{k} \backslash 3 P_{1}^{k}\right) \cap \Omega_{k}, \ldots, E_{j}^{k}=\left(3 P_{j}^{k}\right)\left(\bigcup_{l=1}^{j-1} 3 P_{l}^{k}\right) \cap \Omega_{k}, \ldots
$$

The sets $E_{j}^{k}$ are pairwise disjoint and $\mathbb{R}^{n}=\bigcup_{k \in \mathbb{Z}} \Omega_{k}=\bigcup_{j, k} E_{j}^{k}$. Fix $L \in \mathbb{N}$ and suppose that $|k| \leq L$. By Lemma 2.1 and (2.2), and since

$$
\exp \left(\frac{1}{\left|Q_{x}^{k}\right|} \int_{Q_{x}^{k}} \log v^{-1 / p}(x) d x\right)<1,
$$

we obtain

$$
\begin{aligned}
I_{L}: & =\int_{\bigcup_{|k| \leq L} \Omega_{k}}\left(G_{\alpha} f(x)\right)^{q} u(x) d x \\
\leq & \sum_{j, k} 2^{(k+1) q} \int_{E_{j}^{k}} u(x) d x \\
< & C \sum_{j, k}\left(\frac{1}{\left|P_{j}^{k}\right|} \int_{P_{j}^{k}} f(x) v^{1 / p}(x) d x\right)^{q}\left(\left|Q_{x}^{k}\right|^{q \alpha / n} \exp \left(\frac{q}{\left|Q_{x}^{k}\right|} \int_{Q_{x}^{k}} \log v^{-1 / p}(x) d x\right)\right) \\
< & C \sum_{j, k}\left(\frac{1}{\left|P_{j}^{k}\right|} \int_{P_{j}^{k}} f(x) v^{1 / p}(x) d x\right)^{q} \\
& \quad \times\left(\left|3 P_{j}^{k}\right|^{q \alpha / n} \exp \left(\frac{q}{\left|3 P_{j}^{k}\right|} \int_{3 P_{j}^{k}} \log v^{-1 / p}(x) d x\right)\right)\left(\int_{E_{j}^{k}} u(x) d x\right) \\
= & C \sum_{j, k}\left(T_{L}\left(f v^{1 / p}\right)(j, k)\right)^{q} \sigma(j, k),
\end{aligned}
$$

where

$$
\sigma(j, k)=\left(\left|3 P_{j}^{k}\right|^{q \alpha / n} \exp \left(\frac{q}{\left|3 P_{j}^{k}\right|} \int_{3 P_{j}^{k}} \log v^{-1 / p}(x) d x\right)\right)\left(\int_{E_{j}^{k}} u(x) d x\right)
$$

and

$$
T_{L} h(j, k)=\frac{1}{\left|P_{j}^{k}\right|} \int_{P_{j}^{k}} h, \quad j \in \mathbb{N}, k \in \mathbb{Z},|k| \leq L .
$$

It is obvious that $T_{L}$ is linear and bounded from $L^{\infty}\left(\mathbb{R}^{n}, d x\right)$ into $L^{\infty}(\mathbb{N} \times \mathbb{Z}, \sigma)$ uniformly on $L$. 
Next, we will prove that $T_{L}$ is bounded from $L^{1}\left(\mathbb{R}^{n}, d x\right)$ into $L^{q / p, \infty}(\mathbb{N} \times \mathbb{Z}, \sigma)$ independently of $L$. Let $\lambda>0$. The dyadic cubes of the collection $\left\{P_{j}^{k}: T_{L} h(j, k)>\lambda\right\}$ have bounded size, since $|k| \leq L$ and for every $k$, the cubes $\left\{P_{j}^{k}\right\}$ have bounded size. Hence we can extract a maximal subcollection $\left\{P_{i}\right\}_{i}$ such that for every $(j, k)$, with $T_{L}(j, k)>\lambda, P_{j}^{k} \subset P_{i}$, for some $i$. The pairwise disjointness of the sets $E_{j}^{k}$ and the fact that $E_{j}^{k} \subset 3 P_{j}^{k}$ give

$$
\begin{aligned}
& \sum_{\left\{(j, k): T_{L} h>\lambda\right\}}\left(\exp \left(\frac{1}{\left|3 P_{j}^{k}\right|} \int_{3 P_{j}^{k}} \log v^{-1 / p}(x) d x\right)\right)^{q} \int_{E_{j}^{k}} u(x) d x \\
& \quad \leq \sum_{\left\{(j, k): T_{L} h>\lambda\right\}} \int_{E_{j}^{k}}\left(G\left(v^{-1 / p} \chi_{3 P_{j}^{k}}\right)\right)^{q} u(x) d x \\
& \quad \leq C \sum_{i} \int_{P_{i}}\left(G\left(v^{-1 / p} \chi_{3 P_{i}}\right)\right)^{q} u(x) d x .
\end{aligned}
$$

By (2.4), the pairwise disjointness of the cubes $\left\{P_{i}\right\}_{i}$ and since $p \leq q$, we get

$$
\begin{aligned}
\sum_{i} \int_{P_{i}}\left(G\left(v^{-1 / p} \chi_{3 P_{i}}\right)\right)^{q} u(x) d x & \leq C \sum_{i}\left|3 P_{i}\right|^{q / p} \\
& <C 3^{n q / p}\left(\frac{1}{\lambda} \sum_{i} \int_{P_{i}} h(x) d x\right)^{q / p} \\
& <C\left(\frac{1}{\lambda} \int_{\bigcup_{i} P_{i}} h(x) d x\right)^{q / p} \\
& \leq C\left(\frac{1}{\lambda} \int_{\mathbb{R}^{n}} h(x) d x\right)^{q / p}
\end{aligned}
$$

which means that $T_{L}$ is bounded from $L^{1}\left(\mathbb{R}^{n}, d x\right)$ into $L^{q / p, \infty}(\mathbb{N} \times \mathbb{Z}, \sigma)$ independently of $L$. By the Marcienkiewich interpolation theorem we have that $T_{L}$ is bounded from $L^{p}\left(\mathbb{R}^{n}, d x\right)$ into $L^{q}(\mathbb{N} \times \mathbb{Z}, \sigma)$, that is,

$$
I_{L} \leq C \sum_{j, k}\left(T_{L}\left(f v^{1 / p}\right)(j, k)\right) \sigma(j, k) \leq C\left(\int_{\mathbb{R}^{n}} f^{p}(x) v(x) d x\right)^{q / p} .
$$

Since $C$ does not depend on $L$ we can let $L \rightarrow \infty$ and we get the desired inequality, and this completes the proof of the theorem.

Definition 2.3 [5]. A weight $v$ on $\mathbb{R}^{n}$ is said to belong to the class $A_{\infty}$ if

$$
\exp \left(\frac{1}{|Q|} \int_{Q} \log v(t) d t\right) \leq C \frac{1}{|Q|} \int_{Q} v(t) d t
$$

for some $C>0$ and any cube $Q$ with sides parallel to the axes. 
The following corollary shows that in the diagonal case the extra condition of Theorem 2.2 is actually not needed.

Corollary 2.4. Let $p>0$. The inequality

$$
\left(\int_{\mathbb{R}^{n}}\left(G_{\alpha}|f(x)|\right)^{p} v(x) d x\right)^{1 / p} \leq C\left(\int_{\mathbb{R}^{n}}|f|^{p}(x) v(x) d x\right)^{1 / p}
$$

holds for all $f \in L^{p}(v)$ and some $C>0$ if and only $v$ belongs to $A_{\infty}$.

Proof. Take $p=q, \alpha=0$ and $u=v$ in Theorem 2.2. As proved in [9, p. 76], in this case, condition (2.4) is equivalent to the condition $A_{\infty}$. Let $P$ and $Q \subset 3 P$ be an arbitrary cube which intersects $P$ and such that $\left(1 / 2^{n}\right)|P| \leq|Q|<|P|$. Clearly $P \subset 5 Q$. Let $v$ be a weight belonging to the class $A_{\infty}$. Since all weights which satisfy the $A_{\infty}$ condition are also doubling,

$$
\begin{aligned}
\exp \left(\frac{1}{|3 P|} \int_{3 P} \log v(x) d x\right) & \leq C \frac{1}{|3 P|} \int_{3 P} v(x) d x \\
& \leq C \frac{1}{|Q|} \int_{5 Q} v(x) d x \leq C \frac{1}{|Q|} \int_{Q} v(x) d x \\
& \leq C \exp \left(\frac{1}{|Q|} \int_{Q} \log v(x) d x\right) \\
& =\exp \left(\frac{1}{|Q|} \int_{Q} \log C v(x) d x\right),
\end{aligned}
$$

that is, condition (2.2) is satisfied. In this, we have also used Jensen's inequality. The constant $C$ is obviously not the same at each occurrence. Hence (2.5) holds. The converse of the corollary is trivial, and the proof is complete.

For the case $q=\infty$ we have the following proposition.

Proposition 2.5. Let $0 \leq \alpha<n$. The following inequality holds:

$$
\left\|G_{\alpha} f\right\|_{L^{\infty}(v)} \leq\|f\|_{L^{n / \alpha}(v)} .
$$

Proof. This is just a consequence of the fact that $G_{\alpha} \leq M_{\alpha}$. If $\alpha=0$ the inequality is trivial. For $\alpha>0$, the boundedness of $M_{\alpha}$ follows by Hölder's inequality with exponent $n / \alpha$.

REMARK 2.6. If $n=1, p=q$ and $\alpha=0$ we get [9, Theorem 1] and if $n=1$ we obtain [3, Theorem 2]. For $n>1, p=q$, and $\alpha=0$ we have [1, Theorem 1.5] with the observation that our a priori condition seems easier to check. In this way we partially answer an open question posed in [1, p. 5].

REMARK 2.7. By the Lebesgue differentiation theorem, if $1 / p-1 / q>\alpha / n$, inequality (2.3) cannot hold unless $u \equiv 0$ a.e. Therefore the only nontrivial case is when $1 / p-1 / q \leq \alpha / n$. For $\alpha=0$ this forces $p=q$. 


\section{Weak-type weighted inequalities}

In this section we study weak-type inequalities for the fractional geometric maximal operator, that is, necessary and sufficient conditions for the weights $u$ and $v$ such that $G_{\alpha}: L^{p}(v) \rightarrow L^{q, \infty}(u)$. In order to prove the main theorem we need some technical results. We start with a very simple and useful covering lemma.

Lemma 3.1. Let $\left\{Q_{j}\right\}_{j \in J}$ be an arbitrary family of cubes in $\mathbb{R}^{n}$ such that $\sup _{j \in J}\left|Q_{j}\right|<\infty$. Then, from this family we can select a subsequence $Q_{1}, Q_{2}, \ldots, Q_{n}, \ldots$ (finite or infinite) of disjoint cubes such that $\bigcup_{j \in J} Q_{j} \subseteq \bigcup_{j \in N} 5 Q_{j}$.

Proof. For the proof see [6, p. 10],

Lemma 3.2. Let $\alpha \in[0, n), 0<p \leq q<\infty$ and let $Q \subset \mathbb{R}^{n}$ be a cube. If

$$
\int_{Q} u(x) d x \leq C\left(|Q|^{1-\alpha p / n} \exp \left(\frac{1}{|Q|} \int_{Q} \log v(x) d x\right)\right)^{q / p},
$$

then

$$
\left(|Q|^{\alpha / n} \exp \left(\frac{1}{|Q|} \int_{Q} \log f(x) d x\right)\right)^{q} \int_{Q} u(x) d x \leq C\left(\int_{Q} f^{p}(x) v(x) d x\right)^{q / p}
$$

for any measurable function $f>0$.

Proof. We may suppose without loss of generality that $f$ is bounded and compactly supported and that $v$ has a positive lower bound (see, for example, [9]). Let $Q \subset \mathbb{R}^{n}$ be a cube. By elementary computations and the integral form of the arithmetic meangeometric mean inequality (see [8]),

$$
\begin{aligned}
\left(|Q|^{\alpha / n}\right. & \left.\exp \left(\frac{1}{|Q|} \int_{Q} \log f(x) d y\right)\right)^{q} \\
& =\left(|Q|^{\alpha / n} \exp \left(\frac{1}{|Q|} \int_{Q} \log f(x) v^{1 / p}(x) v^{-1 / p}(x) d x\right)\right)^{q} \\
& =|Q|^{q \alpha / n}\left(\exp \left(\frac{1}{|Q|} \int_{Q} \log f(x) v^{1 / p}(x) d x\right)\right)^{q}\left(\exp \left(\frac{1}{|Q|} \int_{Q} \log v^{-1 / p}(x) d x\right)\right)^{q} \\
& =|Q|^{q \alpha / n}\left(\exp \left(\frac{1}{|Q|} \int_{Q} \log f^{p}(x) v(x) d x\right)\right)^{q / p}\left(\exp \left(\frac{1}{|Q|} \int_{Q} \log v(x) d x\right)\right)^{-q / p} \\
& \leq|Q|^{\mid \alpha / n}\left(\frac{1}{|Q|} \int_{Q} f^{p}(x) v(x) d x\right)^{q / p}\left(\exp \left(\frac{1}{|Q|} \int_{Q} \log v(x) d x\right)\right)^{-q / p} .
\end{aligned}
$$

Multiplying now both sides by $\int_{Q} u(x) d x$ and taking into account our hypothesis, we get the desired inequality.

We are now ready to prove the main theorem of this section, concerning the weak boundedness of the geometric fractional maximal operator $G_{\alpha}$. 
Theorem 3.3. Let $\alpha \in[0, n)$ and $0<p \leq q<\infty$. Suppose that $u$ is a doubling weight. Then the inequality

$$
\int_{\left\{G_{\alpha} f>\lambda\right\}} u(x) d x \leq C \lambda^{-q}\left(\int_{\mathbb{R}^{n}} f^{p}(x) v(x) d x\right)^{q / p}
$$

holds, for any measurable function $f \in L^{p}(v)$ and any $\lambda>0$, if and only if

$$
\int_{Q} u(y) d y \leq C\left(|Q|^{1-\alpha p / n} \exp \left(\frac{1}{|Q|} \int_{Q} \log v(x) d x\right)\right)^{q / p},
$$

for any cube $Q \subset \mathbb{R}^{n}$, with sides parallel to the coordinate axes.

Proof. We prove first the sufficiency of (3.1) for (3.2). By the monotone convergence theorem it is enough to prove (3.1) for bounded functions with compact support. Without loss of generality we may also assume that $v$ has a positive lower bound (see, for example, [9]). We assume that supp $f \subset B(0, R), R>0$. For bounded functions with compact support the fractional maximal function satisfies

$$
M_{\alpha} f(x) \approx|x|^{\alpha-n} \text { for }|x|>R .
$$

Hence the set $\left\{x: M_{\alpha} f(x)>\lambda\right\}$ is bounded for a fixed $\lambda$. Since $G_{\alpha} f(x) \leq M_{\alpha} f(x)$, the set

$$
E_{\lambda}:=\left\{x: G_{\alpha} f(x)>\lambda\right\}
$$

is bounded. By the definition of the geometric fractional maximal operator, for each $x \in E_{\lambda}$ there exists a cube $Q_{x}$ which contains $x$ and such that

$$
\left|Q_{x}\right|^{\alpha / n} \exp \left(\frac{1}{\left|Q_{x}\right|} \int_{Q_{x}} \log f(t) d t\right)>\lambda .
$$

Obviously $E_{\lambda} \subset \cup_{x} Q_{x}$. By the arithmetic mean-geometric mean inequality,

$$
\left|Q_{x}\right|^{\alpha / n} \frac{1}{\left|Q_{x}\right|} \int_{Q_{x}} f(t) d t \geq|Q|^{\alpha / n} \exp \left(\frac{1}{\left|Q_{x}\right|} \int_{Q_{x}} \log f(t) d t\right)>\lambda
$$

and thus $\sup _{x}\left|Q_{x}\right|<\lambda^{n / \alpha-n} R^{n / n-\alpha}<\infty$, that is, the cubes $\left\{Q_{x}\right\}_{x}$ have bounded size. By Lemma 3.1 we can choose a disjoint subsequence $Q_{1}, Q_{2}, Q_{3}, \ldots$ such that $E_{\lambda} \subset$ $\bigcup_{j \in \mathbb{N}} 5 Q_{j}$. Hence by (3.2), Lemma 3.2 and the assumption on $u$,

$$
\begin{aligned}
\int_{\left\{x: G_{\alpha} f(x)>\lambda\right\}} u(x) d x & \leq \int_{\cup_{j \in \mathbb{N}} 5 Q_{j}} u(x) d x \leq \sum_{j \in \mathbb{N}} \int_{Q_{j}} u(x) d x \\
& \leq C \sum_{j \in \mathbb{N}}\left(\left|Q_{j}\right|^{\alpha / n} \exp \left(\frac{1}{\left|Q_{j}\right|} \int_{Q_{j}} \log f(x) d x\right)\right)^{-q}\left(\int_{Q_{j}} f^{p}(x) v(x) d x\right)^{q / p} \\
& <C \sum_{j \in \mathbb{N}} \lambda^{-q}\left(\int_{Q_{j}} f^{p}(x) v(x)\right)^{q / p} \\
& \leq C^{q} \lambda^{-q}\left(\sum_{j \in \mathbb{N}} \int_{Q_{j}} f^{p}(x) v(x) d x\right)^{q / p} \leq C \lambda^{-q}\left(\int_{\mathbb{R}^{n}} f^{p}(x) v(x) d x\right)^{q / p},
\end{aligned}
$$


since $q / p \geq 1$ and the cubes $Q_{j}$ are disjoint. By a classical limiting argument, the inequality above holds for all $f \in L^{p}(v)$. This proves the first part of the theorem.

Conversely, for a fixed cube $Q$, take

$$
\lambda=\frac{1}{2}|Q|^{\alpha / n}\left(\exp \left(\frac{1}{|Q|} \int_{Q} \log v(x) d x\right)\right)^{-1 / p}
$$

and $f(x)=\chi_{Q}(x) v^{-1 / p}(x)$. Then

$$
G_{\alpha}\left(\chi_{Q} v^{-1 / p}\right)(x) \geq|Q|^{\alpha / n}\left(\exp \left(\frac{1}{|Q|} \int_{Q} \log v(x) d x\right)\right)^{-1 / p}>\lambda
$$

for all $x \in Q$, and hence $Q \subset\left\{x: G_{\alpha}\left(\chi_{Q} v^{-1 / p}\right)(x)>\lambda\right\}$. Consequently, we can derive (3.2) from (3.1), and the proof of the theorem is complete.

In the following corollary we prove the diagonal case.

Corollary 3.4. Let $p>0$. The inequality

$$
\int_{\left\{G_{\alpha} f>\lambda\right\}} v(x) d x \leq C \lambda^{-p}\left(\int_{\mathbb{R}^{n}} f^{p}(x) v(x) d x\right)
$$

holds, for any measurable function $f \in L^{p}(v)$ and for any $\lambda>0$, if and only if $v$ belongs to $A_{\infty}$.

Proof. Take $p=q, \alpha=0$ and $u=v$ in Theorem 3.3. Since any weight from the class $A_{\infty}$ is doubling, the a priori condition of Theorem 3.3 is automatically satisfied. The reversed part of the corollary is trivial, and the proof is complete.

REMARK 3.5. A consequence of the two corollaries is the known fact that the weak and strong cases for the geometrical maximal operator are equivalent (see for example, [9]). If $n=1, p=q, \alpha=0$ we get [9, Theorem 2]. If $n=1$ we obtain [3, Theorem 2]. If $n>1, p=q, \alpha=0$ we get [1, Theorem 1.4]. The above theorem generalises these results to a larger range of parameters, without assuming extra doubling conditions on weights. Thus our theorem answers some open questions raised in [1].

Remark 3.6. As shown in [9, Section 6] there exist $u, v$ satisfying the strong condition (2.4) but not the weak condition (3.1). By the Lebesgue differentiation theorem, if $1 / p-1 / q-\alpha / n>0$, inequalities (3.2) and (2.3) cannot hold unless $u \equiv 0$ a.e. Thus the only interesting case is when $1 / p-1 / q-\alpha / n \leq 0$. For $\alpha=0$, this restricts to $p=q$.

\section{Acknowledgements}

The first named author would like to thank the Applied Models group of Karlstad University and especially Professor Claes Uggla, for support which made this research possible. We would like to thank the referee for useful remarks which have improved the final version of this paper. 


\section{References}

[1] D. Cruz-Uribe, 'The minimal operator and the geometric maximal operator in $\mathbb{R}^{n}$ ', Studia Math. 144 (2001), 1-37.

[2] D. Cruz-Uribe and C. J. Neugebauer, 'Weighted norm inequalities for the geometric maximal operator', Publ. Mat. 42 (1998), 239-263.

[3] D. Cruz-Uribe, C. J. Neugebauer and V. Olesen, 'Weighted norm inequalities for geometric fractional maximal operators', J. Fourier Anal. Appl. 5 (1999), 45-66.

[4] J. Garcia-Cuerva and J. M. Martell, 'Two-weight norm inequalities for maximal operators and fractional integrals on nonhomogeneous spaces', Indiana Univ. Math. J. 50 (2001), 1241-1280.

[5] L. Grafakos, Classical and Modern Fourier Analysis (Pearson Education, Upper Saddle River, NJ, 2004).

[6] E. M. Stein, Singular Integrals and Differentiability Properties of Functions (Princeton University Press, Princeton, NJ, 1970).

[7] E. Sawyer, 'A characterization of a two-weight norm inequality for maximal operators', Studia Math. 144 (1982), 1-11.

[8] W. Rudin, Real and Complex Analysis, 3rd edn (McGraw-Hill, New York-St Louis-San Fransisco, 1986).

[9] X. Yin and B. Muckenhoupt, 'Weighted inequalities for the maximal geometric mean operator', Proc. Amer. Math. Soc. 124 (1996), 75-81.

SORINA BARZA, Department of Mathematics, Karlstad University, S-65188 Karlstad, Sweden

e-mail: sorina.barza@kau.se

CONSTANTIN P. NICULESCU, Department of Mathematics, University of Craiova, Street A.I. Cuza 13, RO-200585 Craiova, Romania e-mail: cniculescu@centralucv.ro 